\title{
High Dynamic Electromechanical Conversion Model for PMSM Drives
}

\author{
S.Bolognani \\ Dept. of Electric Engineering \\ University of Padova \\ Padova, Italy \\ bolognani@die.unipd.it
}

\author{
L.Peretti, M.Zigliotto \\ Dept. of Engineering and Management \\ University of Padova \\ Vicenza, Italy \\ luca.peretti@unipd.it, mauro.zigliotto@unipd.it
}

\begin{abstract}
Several emerging applications require fast and precise torque control. Torque measurements are still expensive, bulky and delicate. On the other hand, estimation techniques are all valid in principle, but their accuracy is largely affected by model identification and commissioning. This paper proposes an accurate model for the electromechanical conversion mechanism in a Permanent Magnet Synchronous Motor drive, based on voltage and current signals. It includes all motor losses, to get an enhanced overall accuracy. The key-feature is the commissioning procedure, based on a set of off-line measurements. The procedure details, as well as the successful experimental results on a real laboratory prototype, are reported in the paper.
\end{abstract}

Keywords- Electric drives; Electromechanical conversion; Torque estimation; Parameter commissioning; PM synchronous motor drives; Brushless motor drives.

\section{INTRODUCTION}

The estimation of the either the electromechanical or shaft torque is arousing living interest in many advanced drives applications. While the study of torque components and their dependence on motor geometry is a key issue for an improved motor design [1], the availability of instantaneous torque estimation is sought both in high-power applications, where the shaft torque transducer is both expensive and difficult to place and maintain, and in sensorless direct torque control as well. For example, in [2] the precise torque value is necessary for a proper ripple compensation in a sensorless Brushless DC motor. The solution is based on the conventional expression of the electromagnetic torque, and it relies on the knowledge of both speed and back-electromotive force, which are obtained by a simplified Kalman filter and sliding mode observer respectively. Similarly, in [3] the instantaneous torque value is obtained by a MRAS technique to reduce torque pulsation due to flux harmonics. The results are effective, with good torque estimation accuracy. Anyway, the estimation does not include neither iron losses, which are not negligible, especially for high-power drives, nor mechanical losses, which are important to derive the net shaft torque.

Another example is reported in [4], where the highly nonlinear electromagnetic flux-current-angle and torque-currentangle relationships of a switched reluctance motor are obtained by a Cerebellar Model Adaptive Control (CMAC), and an adaptive B-Spline network gives a real-time torque estimation. For high speed operation however, CMAC requires a FPGA hardware implementation, and the B-spline needs a TMS320C6711 DSP as a co-processor for a TMS320F243 controller. Therefore, the computational requirements are heavy, and the estimate still suffers of the accuracy of the motor electromagnetic model. A fair example of torque estimate for induction motors is reported in [5]. The algorithm is based on stator voltage and machine current measurements. The stator flux synthesis, which leads to the torque estimation, presents the tricky problem of the offset in the time integral. The digital implementation is performed by a Programmable Cascaded Low-Pass Filters [6], implemented by using Recurrent Neural Network (RNN-PCLPF) trained by an algorithm based on Kalman filter. The precision falls within the $2 \%$, even if iron and additional losses are not included, which are generally not negligible, especially in large motor drives.

Latest research strategies, successfully applied to the torque estimation of internal combustion engines, are based on the stochastic approach and described in [7] and [8]. This approach, which is based on the correlations between incylinder pressure and crankshaft speed variations, gives accurate estimations of the instantaneous in-cylinder pressure, with light computational requirements. It is believed that the method may have very high potential also for application in electrical motors.

Anyway, and despite the well-motivated interest of industry about the topic, evidently the ultimate solution hasn't been proposed yet. The precise electromechanical conversion model plays a crucial rule in the torque estimate, and it involves manifold aspects, like the specific motor and drive design, available measurements, signal accuracy, numerical precision and computational requirements. For all that, it is acceptable that a precise model may be customised on the application. Nevertheless, to our experience, an accuracy below 3-4\%, referred to the nominal torque, on wide speed and load ranges, is still a charming challenge in many cases.

This paper proposes a simple and precise model for electromechanical conversion in permanent magnet synchronous motor (PMSM) drives. It relies on both phase currents and voltages signals, which are both very delicate issues. A scheme for precise inverter voltage non-linearity compensation has been specifically developed and tested on the field.

As detailed in the paper, the estimate is carried out without any magnetic model of the motor, which is not handy and does not assure enough precision, especially for high power drives. Particular emphasis is given to the innovative commissioning procedure, which is performed by a series of standard measurements followed by a specific data post-processing path. The paper includes experimental results, which confirm the effectiveness of the procedure.

The accuracy of the electromechanical conversion model has been finally verified by comparison with a wide bandwidth, high precision torsiometer, coupled to the motor shaft. 


\section{THE Motor ModeL}

Conventional vector control strategies are performed under the assumption that there are no iron losses in the motor. However, since the iron loss influences both the flux linkage and electromagnetic torque, they have been necessarily included in the motor model. Several authors have made an attempt to consider the iron loss in vector-controlled ac motor drives [9-11]. In those studies, iron losses are modelled as the thermal power dissipated on a "iron loss" resistance placed in series, or in parallel, to the conventional PMSM equivalent circuits. From a modelling point of view, it has been found that there is substantial equivalence between series and parallel types [12]. Hereafter, the latter will be chosen for its better adherence to the physical meaning [12]. The PMSM voltage balance equation, in a stationary $\alpha-\beta$ reference frame are the following:

$$
\begin{aligned}
& u_{\alpha}=R_{s} i_{\alpha}+\frac{d \lambda_{\alpha}}{d t} \\
& u_{\beta}=R_{s} i_{\beta}+\frac{d \lambda_{\beta}}{d t}
\end{aligned}
$$

where $R_{s}$ is the stator resistance and $\lambda_{\alpha, \beta}$ are the flux linkages. The related PMSM equivalent circuits are reported in Figure 1 .

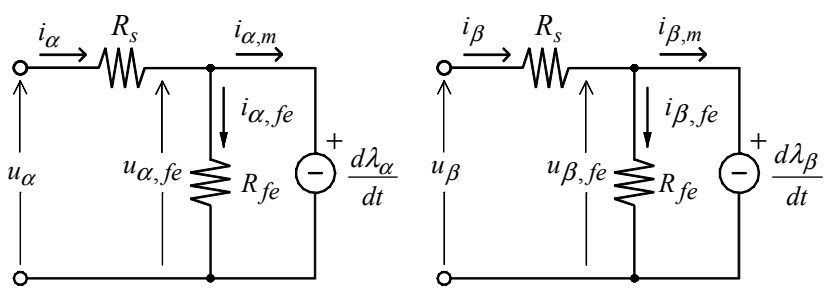

Figure 1. PMSM $\alpha$ - $\beta$ equivalent circuits, including iron losses.

An iron loss resistance $R_{f e}$ is inserted in parallel with each magnetizing branch. Actually, the axis currents $\left(i_{\alpha}, i_{\beta}\right)$ are divided into iron loss currents $\left(i_{\alpha, f e}, i_{\beta, f e}\right)$ and magnetizing currents $\left(i_{\alpha, m}, i_{\beta, m}\right)$ :

$$
\begin{aligned}
& i_{\alpha, f e}=G_{f e} \frac{d \lambda_{\alpha}}{d t} \\
& i_{\beta, f e}=G_{f e} \frac{d \lambda_{\beta}}{d t}
\end{aligned}
$$

where $G_{f e}=1 / R_{f e}$ is the iron loss conductance, which is in general a non-linear function of electrical angular velocity $\omega_{m e}$ [12].

\section{ElECTROMECHANICAL CONVERSION MODEL}

From the model outlined in the previous section, it is clear that since both the copper losses and the iron losses have been explicitly considered, the electromagnetic torque can be obtained by the cross-product of the direct and quadrature flux linkages by the net stator currents:

$$
\tau_{e}=\frac{3}{2} p\left(\lambda_{\alpha} i_{\beta, m}-\lambda_{\beta} i_{\alpha, m}\right)
$$

Expressions (1)-(3) describe the electromechanical conversion model, which is outlined in Figure 2.

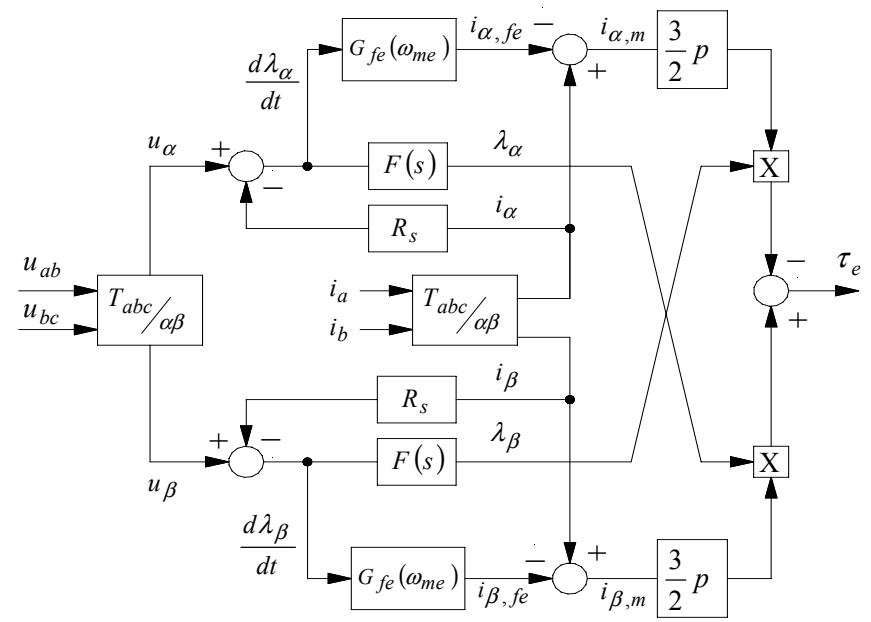

Figure 2. Block schematic of the electromechanical conversion model.

$F(s)$ represent a special integration block, whose details will be given in the next section, while $T_{a b c / \alpha \beta}$ is the conventional three-to-two phases stationary coordinates transformation.

The proposed model accounts for any saturation effect, without the need of customised look-up tables, overcoming one of the heaviest weaknesses of conventional models. It is worth to note that despite its simplicity, the accuracy directly depends upon two key-elements, namely the voltage signal accuracy and the flux linkage integration, which will be addressed later in the paper. The model estimates electromechanical torque, which can lead the shaft torque by considering both the mechanical and additional torque losses.

\section{A. Motor voltages acquisition.}

The motor voltages can be either measured or estimated from the voltage references. As the direct measure is usually discarded, due to the PWM nature of the motor phase voltages, the latter seems a more viable choice. To maintain the necessary precision, the difference between references and actual voltage, due to the inverter non-linearities has been drastically reduced by an innovative compensation technique based on repetitive control $[13,16]$.

The key feature lies in the periodical nature of the voltage distortion, which can be profitably expressed in a synchronous $d q$ rotating frame, locked to the phase $\vartheta_{i}$ of the current space vector, as:

$$
\begin{aligned}
& u_{\text {dist }, d}\left(\vartheta_{i}\right)=\frac{4 t_{d}}{\pi T_{c}} U_{d c}\left[1-\sum_{n=1}^{+\infty}(-1)^{n} \frac{2}{36 n^{2}-1} \cos \left(6 n \vartheta_{i}\right)\right] \\
& u_{\text {dist }, q}\left(\vartheta_{i}\right)=\frac{4 t_{d}}{\pi T_{c}} U_{d c} \sum_{n=1}^{+\infty}(-1)^{n} \frac{12 n}{36 n^{2}-1} \sin \left(6 n \vartheta_{i}\right)
\end{aligned}
$$

where $T_{c}$ is the PWM switching period, $U_{d c}$ is the $d c$-link voltage, and $t_{d}$ is equal to:

$$
t_{d}=t_{\text {off }}-t_{\text {on }}-t_{d s}
$$


where $t_{\text {off }}$ and $t_{\text {on }}$ are the fall and rise times of the inverter power switches, and $t_{d s}$ is the safe interval between the commutation edges of the upper and lower devices in the inverter leg. Aside from the constant term in $u_{\text {dist }, d}$, the distortion can be identified as a sum of $6 n$-th harmonic sinusoidal components $(n=1, \ldots,+\infty)$. Also the effect of parasitic capacitances of IGBTs, during the commutation at low current levels, can be viewed as a periodical disturbance. Since there are six zero crossings during a $2 \pi$ rotation of a synchronous frame, the related distortion effect is well approximated by a sum of $6 n$-th harmonics $(n=1, \ldots,+\infty)$, which overlap the harmonics coming from (4).

In this work, the repetitive control is exploited to generate an application-specific look-up table (LUT) during a first selfcommissioning phase. The generated compensation voltage for the natural $a b c$ reference frame are stored into three look-up tables, which will substitute the conventional step-like compensation techniques at low current levels. For higher current levels, LUTs are merged with the conventional steplike compensation, and then used in the whole speed range. This algorithm improves the correspondence between reference voltage and actual values, so that the formers can be profitably used in the proposed electromechanical conversion model.

As example, Figure 3 shows a comparison between reference and actual voltages in case of no compensation, classical step-like compensation and used compensation at a speed of 0.015 p.u.
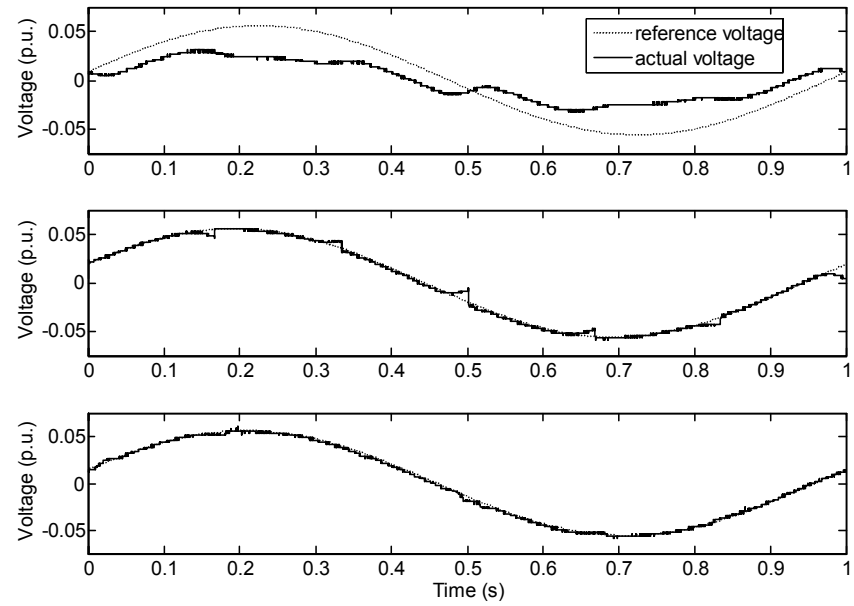

Figure 3. Reference and actual voltages. Up: without any compensation. Middle: conventional compensation. Bottom: proposed LUT-based technique.

It is worth to note that the proposed technique preserves the low-frequency content of the voltage signal, while deleting the contribution at the switching frequency and its harmonics. The same holds for the phase currents, which are sampled synchronously with the switching period, to skip aliasing effects. Thus, actual voltages and currents do not fit for direct derivation of PWM losses, which are sometimes non negligible, as discussed in [14] and reported later in the paper.

\section{B. Flux linkage integration.}

In principle, the flux linkages are calculated by pure integration of their own derivatives, obtained from the phase voltages (1). The unavoidable presence of input offset makes this solution unfeasible. An acceptable trade-off between algorithm complexity and effectiveness was found to be a cascade of a low-pass filter and a high-pass filter, which gives a overall transfer function

$$
F(s)=\frac{s \tau^{2}}{(1+s \tau)^{2}}
$$

Figure 4 reports the Bode plot of $F(s)$. The proposed function approximates the ideal integrator for sufficiently high frequencies. As well, the torque observer will work properly above that limit, which was considered acceptable. Anyway, when low-speed torque estimation is required, a modified integrator with adaptive compensation [15] should be used instead, at the cost of a slightly increased system complexity.

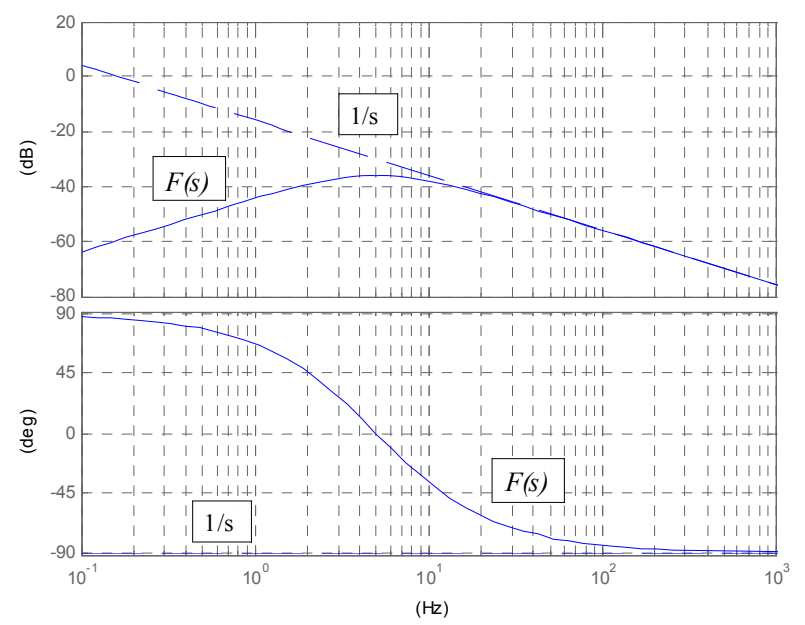

Figure 4. Bode diagram of pure integrator and proposed implementation.

To increase integration accuracy, the phase and magnitude distortions introduced by $F(s)$, as function of frequency, can been stored in look-up table and properly compensated on-line.

\section{The COMmissioning PRocedure}

The commissioning procedure plays a crucial rule in the successful tuning of the electromechanical conversion model of Figure 2. Figure 5 reports a sketch of the experimental setup.

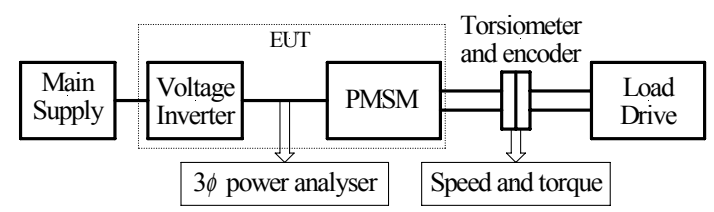

Figure 5. Experimental setup for preliminary power measurements.

The procedure consists of two main steps, both performed off-line. The first one is a series of power measurements, under specified working conditions. The PMSM input power $P_{\text {in }}$ was measured by a PPA 2500 KinetiQ power analyser, which is especially suitable for on-site use. It gives fundamental and rms currents and voltages with high accuracy and calculates active, reactive and apparent power and other derived values. Both fundamental line-to-line voltages and phase currents were measured directly, without any external probe. The 
instrument's basic accuracy is of $0.04 \%$ and its bandwidth of $0.01 \mathrm{~Hz}$ to $2 \mathrm{MHz}$. The load is represented by a second drive (Figure 5) that can be either speed or torque regulated.

The second step of the proposed commissioning procedure is a post-elaboration of the collected data, according to a sequence that is committed to extract the desired parameters and their dependence upon operating conditions.

\section{A. Power measurements.}

The first batch of measurements was obtained at constant speed and current amplitudes, for different torque levels. The mechanical speed $\omega_{m}$ was imposed by the load drive (Figure $5)$, set in constant speed mode. Actually, the drive under test $(E U T)$ is operated in braking mode, and negative torque values $(\tau<0)$ are expected.

The experiments were then repeated at different speed. Table I reports an example of p.u. data.

TABLE I

MEASUREMENTS AT CONSTANT SPEED AND CURRENT (P.U. VALUES)

\begin{tabular}{|c|c|c|c|c|}
\hline Torque $\tau$ & -0.508 & -0.772 & -0.814 & -0.817 \\
\hline Input Power $P_{\text {in }}$ & -0.292 & -0.447 & -0.472 & -0.473 \\
\hline Line-to-line voltage & 0.294 & 0.450 & 0.505 & 0.549 \\
\hline Total losses $P_{L}$ & 0.013 & 0.016 & 0.016 & 0.017 \\
\hline
\end{tabular}

For each measurement, the total power loss was calculated as the difference between the input power and the mechanical power at the motor shaft:

$$
P_{L}=\left|P_{i n}-\omega_{m} \tau\right|
$$

A second batch was performed at constant speed and flux linkage. The latter was evaluated from the integration of the back electromotive force (bemf), which is a part of the model, as it appears from Figure 1. Table II reports an example of p.u. data collected at nominal flux linkage.

TABLE II

MEASUREMENTS AT CONSTANT SPEED AND FLUX LiNKAGE (P.U. VALUES)

\begin{tabular}{|c|c|c|c|c|}
\hline Torque $\tau$ & -0.468 & -0.578 & -0.666 & -0.806 \\
\hline Input Power $P_{\text {in }}$ & -0.273 & -0.337 & -0.388 & -0.468 \\
\hline Phase current $I$ & 0.468 & 0.582 & 0.677 & 0.833 \\
\hline Total losses $P_{L}$ & 0.008 & 0.010 & 0.012 & 0.016 \\
\hline
\end{tabular}

Once again, the measurements were repeated for different speed, up to the nominal one. The collected data were then post-processed to get, in sequence, the complete model commissioning.

\section{B. Evaluation of the stator resistance.}

The measure of the stator resistance with conventional instrumentation is sometimes unpractical. Here a viable procedure is proposed instead. At constant flux linkage the iron losses are supposed to be constant. The slope of the linear interpolation of the power losses as function of the square current thus returns a resistance value. The slopes, i.e. the resistances, are then plotted as function of speed. It has been found that a linear dependence is obtained by considering the square root of the speed, as reported in Figure 6.

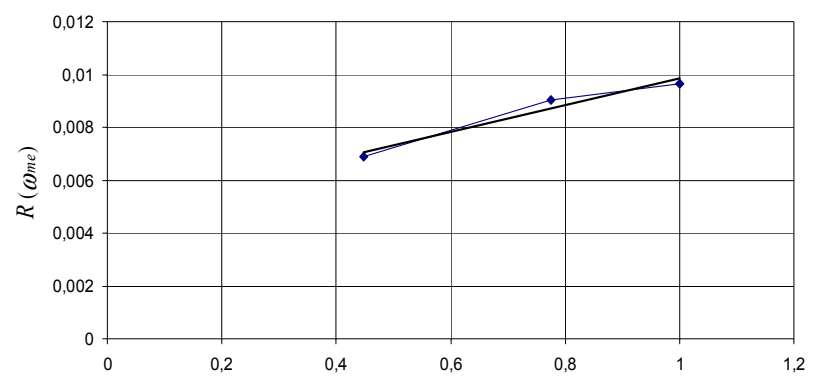

Figure 6. Stator resistance vs. square root of the speed (p.u. values).

The equation of the interpolating straight line is

$$
R\left(\omega_{m e}\right)=k_{1} \sqrt{\omega_{m e}}+R(0)=k_{1} \sqrt{\omega_{m e}}+R_{s}
$$

The intercept $R(0)$ is taken as (constant) stator resistance. The remaining speed-dependant losses are mainly due to the asymmetrical distribution of the current in the stator winding paths, and they will be considered later on, as part of the additional losses.

\section{Iron losses model commissioning.}

According to the schemes of Figure 1, the iron losses can be modelled as the power dissipated on the conductance $G_{f e}\left(\omega_{m e}\right)$.

The commissioning procedure exploits the data obtained at constant speed and current (Table I), so that copper, mechanical and additional losses can be considered altogether as a constant $P_{L, 0}$. The total losses can be expressed as the sum of $P_{L, 0}$ and a term that is a linear function of the squares of the $r m s$ voltages $u_{i, \alpha}, u_{i, \beta}$ (Figure 1):

$$
\begin{aligned}
P_{L, i}\left(\omega_{m e}\right) & =G_{f e}\left(\omega_{m e}\right)\left(u_{i, \alpha e f f}^{2}+u_{i, \beta e f f}^{2}\right)+P_{L, 0}= \\
& =G_{f e}\left(\omega_{m e}\right)\left|u_{i}\right|^{2}+P_{L, 0}
\end{aligned}
$$

where $\left|u_{i}\right|$ is the rms value of the phase voltages, which can be derived from the measurements of Table I, completed with the information about the actual phase currents, readily available from the current control system of the drive.

Figure 7 reports the iron losses (p.u.) as function of the square voltage $\left|u_{i}\right|^{2}$, at three different speeds. As said, the angular coefficients of the straight lines represent the conductance $G_{f e}\left(\omega_{m e}\right)$. Then, the dependence of $G_{f e}\left(\omega_{m e}\right)$ by the speed is easily derived by plotting $G_{f e}\left(\omega_{m e}\right)$ as function of the reciprocal of the speed itself (Figure 8). In this case, it is simply a straight line with slope $m_{f e}$, so that

$$
G_{f e}\left(\omega_{m e}\right)=\frac{m_{f e}}{\omega_{m e}}
$$

with $m_{f e}$ easily derivable from Figure 6. 

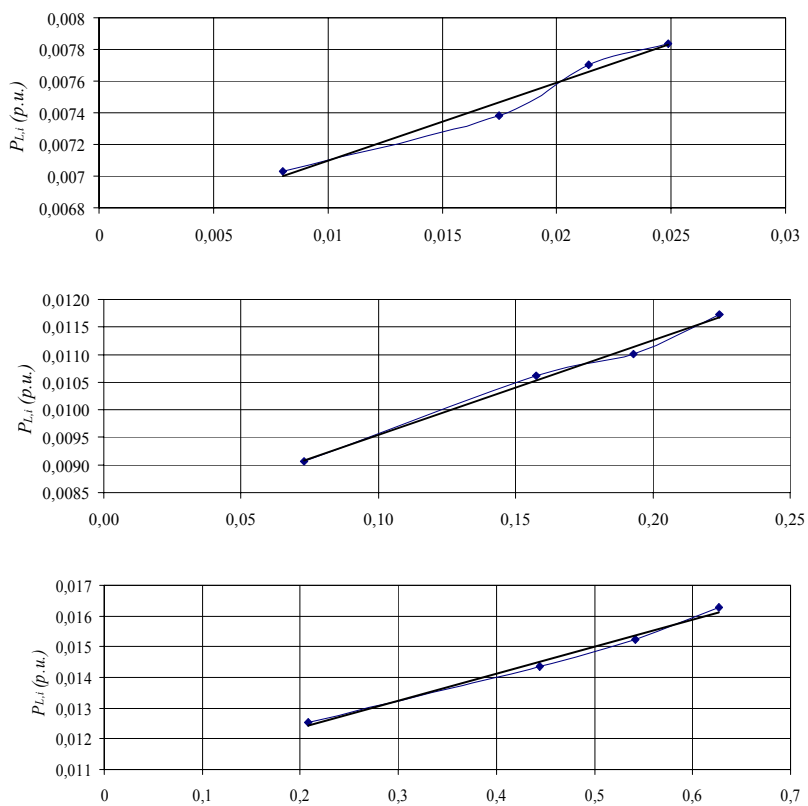

Figure 7. Total losses $P_{L, i}$ as function of square voltage (p.u.). (Up: $\omega_{m}=0.2$ p.u.; Middle: $\omega_{m}=0.6$ p.u. Bottom: $\omega_{m}=1$ p.u.)

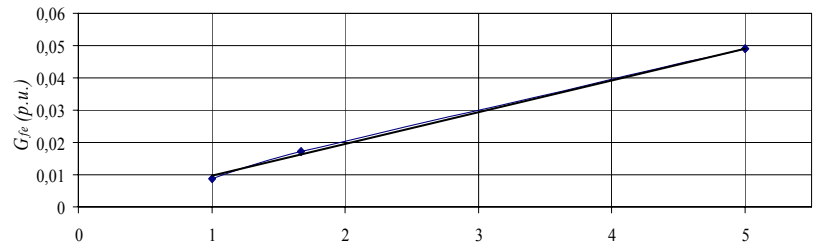

Figure 8. Iron losses conductance $G_{f e}$ as function of speed reciprocal (p.u.)

\section{Additional losses model commissioning.}

Additional losses causes are manifold, and conceptually they cannot be attributed to a single source. Additional losses include, but are not limited to, both PWM losses and skin effects.

The skin effect conductor losses vary with the square root of frequency. For practical PWM current ripples of $20-30 \%$ of rated current, copper loss increase due to PWM, even accounting for skin-effect, is estimated around $10-15 \%$, that alone cannot explain significant total PWM loss. It means that PWM core loss is a dominating mechanism. For PM machines, PWM eddy current losses are supposed to take place mostly in PWM excited armature yoke, because of relatively large equivalent air gap. Some PWM losses are also expected in permanent magnet alloy materials that, as opposed to ferrites, have relatively high conductivity. Accurate and detailed studies have been reported by different Authors, for example [14].

This paper adopts a different approach. Additional losses are not tentatively evaluated from equations based on the probable physical origin of each loss term. Instead, joined additional and mechanical losses $\left(P_{a}+P_{\text {mech }}\right)$ are derived as the difference between total measured losses $P_{L}$ and both iron and copper losses $\left(P_{L, i}\right.$ and $P_{L, c u}$, respectively).

At constant speed and flux (Table II), constant iron losses can be assumed. $P_{a}+P_{\text {mech }}$ are plotted as function of the square amplitude of the phase currents. Figure 9 reports the plot for $\omega_{m}=0.6 p \cdot u$.

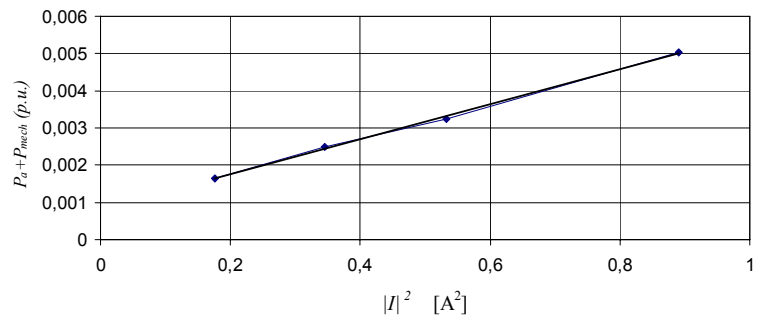

Figure 9. Mechanical and additional losses as function of square current, at constant speed and flux linkage (p.u. values).

Similar plots have been drawn at different speed, resulting in as much again straight lines, each with a specific slope and intercept. The intercepts represent the losses at no-current, which can be liken to mechanical losses. It is possible to elaborate a polynomial approximation of the mechanical losses as function of the speed. In the present work, it has been found that the mathematical link between mechanical losses and speed is well defined by the expression

$$
P_{m e c h}\left(\omega_{m e}\right)=k_{1} \omega_{m e}^{4}+k_{2} \omega_{m e}^{2}
$$

where $k_{1}$ and $k_{2}$ are constant coefficients. The remaining part of $\left(P_{a}+P_{\text {mech }}\right)$ is actually the contribution of additional losses, which is in general a non-linear function of both the current level and the frequency (speed).

Different approaches can be adopted to model the additional losses. A possible way is to derive the additional losses in an adequate number of working points, and store the results in look-up tables (LUTs). Of course, the accuracy is affected by LUTs resolution and their capability of covering the whole drive working conditions. A valid alternative is to investigate a possible explicit relation between additional losses and both speed and current. For example, in the laboratory prototype it has been found that the plot between $P_{a}$ divided by the square current amplitude (i.e. the slopes of the plots like that of Figure 9) and the reciprocal of the square root of the speed, is a straight line, as shown in Figure 10.

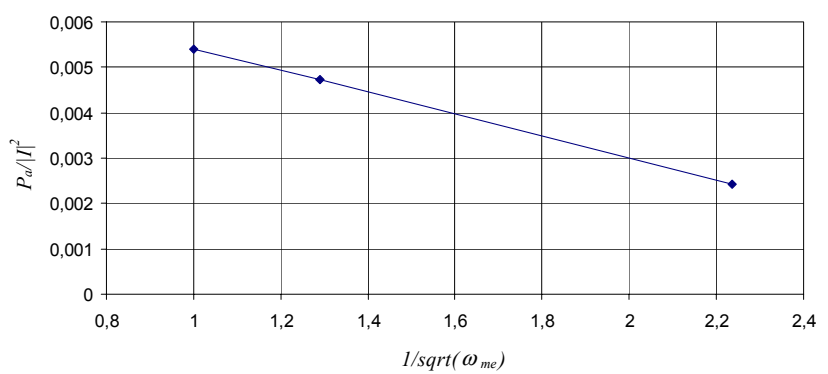

Figure 10. Additional losses as function of speed and current, p.u. values.

The non-linear function that has been used to model additional losses is therefore

$$
P_{a}=\left(\frac{k_{3}}{\sqrt{\omega_{m e}}}+k_{4}\right)|I|^{2}
$$


where $k_{3}$ and $k_{4}$ are constants that can be directly derived from Figure 10.

The additional and mechanical losses are turned into a torque loss by dividing them by the mechanical speed. The torque loss is then properly subtracted to the estimated electromechanical torque $\tau_{e}(3)$, to get the estimation of the net shaft torque $\tau_{s}$ :

$$
\tau_{s}=\tau_{e}-p\left(k_{1} \omega_{m e}^{3}+k_{2} \omega_{m e}+k_{3}|I|^{2} \omega_{m e}^{-3 / 2}+k_{4}|I|^{2} \omega_{m e}^{-1}\right)
$$

where $p=\omega_{m e} / \omega_{m}$ is the number of pole pairs of the PMSM.

\section{EXPERIMENTAL RESULTS}

The electromechanical conversion model of Figure 1 has been implemented on a fast control prototype (FCP) system. The commissioning has been obtained by the procedure outlined in this paper. A first extended batch of measures has lead to the definition of both stator resistance and iron losses conductance. The shaft torque has been measured by a highprecision torquemeter, and compared with the torque estimated by the model (12). Figure 11 reports the relative estimation error, as function of the motor speed, for different load conditions.

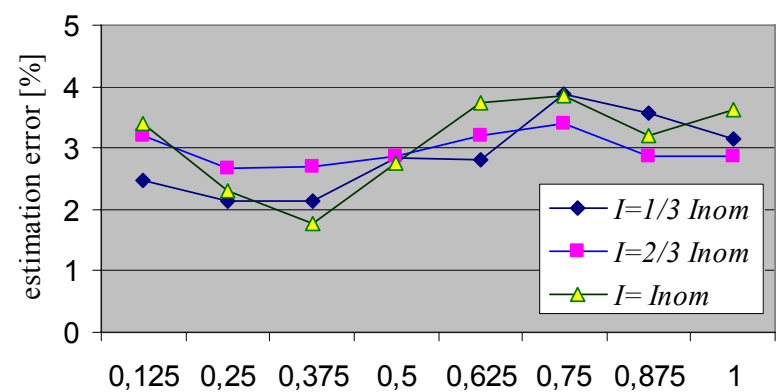

Figure 11. Torque estimation relative error vs. speed (x-axis, p.u.) and current.

It is worth to note that the error remains within $4 \%$ in the whole speed and torque range of the drive. The error is relative to the actual measured torque, thus the reference to the nominal torque value would still improve the figures. The error is always of the same sign, which suggests the possible presence of a systematic error, which will be further investigated.

While accounting for all possible losses, and thus aiming to high accuracy estimation, the proposed electromagnetic conversion model is based on instantaneous voltage estimation and current measurements. After commissioning, it features a bandwidth of about $800 \mathrm{~Hz}$.

\section{CONCLUSIONS}

The paper reports a precise electromechanical conversion model for PMSM drives. Particular emphasis is put on its commissioning, based on input power measurements. The model includes iron and additional losses. The model enables a precise torque estimation, which has been validated by laboratory shaft torque measurements, which have confirmed the good accuracy and excellent dynamic performance of the proposed model.

\section{REFERENCES}

[1] D.M.Ionel, M.Popescu, M.I.McGilp, T. J. E. Miller, S.J.Dellinger, "Assessment of Torque Components in Brushless Permanent-Magnet Machines Through Numerical Analysis of the Electromagnetic Field", IEEE Trans. on IA, Vol.41, No.5, pp.1149-1158, Sept/Oct 2005.

[2] Y.Liu, Z.Q.Zhu, D.Howe, "Instantaneous Torque Estimation in Sensorless Direct-Torque-Controlled Brushless DC Motors", IEEE Transactions on IA, Vol.42, No.5, pp.1275-1283, Sept/Oct. 2006.

[3] S-K. Chung, C-S. Kim, C-G.Kim, M-J. Youn, "A New Instantaneous Torque Control of PM Synchronous Motor for High-Performance Direct-Drive Applications“, IEEE Trans. Power Appl., Vol.13, No.3, pp.388-400, May 1998.

[4] D.S.Reay, "CMAC and B-spline Neural Networks Applied to Switched Reluctance Motor Torque Estimation and Control", 29th Annual Conference of the IEEE Industrial Electronics Society, IECON'03, ISBN:0-7803-7907-1, pp.323-328, 2003.

[5] F.Valias de Carvalho, L.E.Borges da Silva, J.O.Pereira Pinto, B.K.Bose, G.L.Torres, "A DSP Based Torque Meter for Induction Motors", 29th Annual Conference of the IEEE Industrial Electronics Society, IECON'03, ISBN:0-7803-7907-1, pp.414-418, 2003.

[6] B. K. Bose and N. R. Patel, "Programmable Cascaded Low-Pass FilterBased Flux Synthesis for Stator Flux-Oriented Vector-Controlled Induction Motor Drive", IEEE Trans. Ind. Electron., vol.44, No.1, pp.140-143, Feb. 1997.

[7] G.Rizzoni, Y.Guezennec, A.Soliman,B.Lee, "Engine control using torque estimation", US Patent 6866024, March 15, 2005.

[8] P. Gyan, S. Ginoux, J. C. Champoussin, Y. Guezennec, "Crankangle Based Torque Estimation: Mechanistic/Stochastic," SAE Technical Paper 2000-01-0559, 2000.

[9] N.Urasaki, T.Senjyu,K.Uezato,"A Novel Calculation Method for Iron Loss Resistance Suitable in Modeling Permanent-Magnet Synchronous Motors", IEEE Trans. Enegy Conv., Vol.18, No.1, pp.41-47, March 2003.

[10] N. Urasaki, T. Senjyu, and K. Uezato, "An accurate modeling for permanent magnet synchronous motor drives," in Proc. APEC 2000, New Orleans, LA, 2000, pp. 387-392.

[11] S. Morimoto, Y. Tong, Y. Takeda, and T. Hirasa, "Loss minimization control of permanent magnet synchronous motor drives," IEEE Trans. Ind. Electron., vol. 41, pp. 511-517, Sept./Oct. 1994.

[12] N.Urasaki, T.Senjyu, K.Uezato, "Relationship of Parallel Model and Series Model for Permanent Magnet Synchronous Motors Taking Iron Loss Into Account”, IEEE Trans. Energy Conv., Vol. 19, No. 2, pp. 265 270, June 2004

[13] M.Steinbuch, "Repetitive Control with Uncertain Period Time", Automatica, Vol.38, pp.2103-2109, 2002.

[14] A.Ruderman, "Electrical Machine PWM Loss Evaluation Basics", Proc. of $4^{\text {th }}$ International Conference on Energy Efficiency in Motor Driven Systems, EEMODS'05, Heidelberg, Germany, 2005.

[15] J.Hu, B.Wu,"New Integration Algorithms for Estimating Motor Flux over a Wide Speed Range", IEEE Trans. Pow. El., Vol.13, No.5, pp.969 977, Sept 1998.

[16] S.Bolognani, L.Peretti, M.Zigliotto, "Inverter Non-Idealities Override by Repetitive Control", Proceedings of IEEE International Machines and Drives Conference, IEMDC'07, IEEE Catalog No.07EX1597C, ISBN 14244-0743-5, pp.71-76, Antalia, Turkey, 2007. 